\title{
Previously Implanted Cardiac Lead
}

National Cancer Institute

\section{Source}

National Cancer Institute. Previously Implanted Cardiac Lead. NCI Thesaurus. Code C100007.

The coronary sinus / left ventricular lead was not implanted because a lead had been previously implanted. (ACC) 